\title{
Energy-Based Controller Design of Stochastic Magnetic Levitation System
}

\author{
Weiwei Sun, ${ }^{1,2}$ Kaili Wang, ${ }^{1}$ Congcong Nie, ${ }^{1}$ and Xuejun Xie ${ }^{1,2}$ \\ ${ }^{1}$ Institute of Automation, Qufu Normal University, Qufu 273165, China \\ ${ }^{2}$ School of Engineering, Qufu Normal University, Rizhao 276826, China \\ Correspondence should be addressed to Weiwei Sun; wwsun@hotmail.com
}

Received 9 March 2017; Revised 15 May 2017; Accepted 18 July 2017; Published 20 August 2017

Academic Editor: Huanqing Wang

Copyright (c) 2017 Weiwei Sun et al. This is an open access article distributed under the Creative Commons Attribution License, which permits unrestricted use, distribution, and reproduction in any medium, provided the original work is properly cited.

\begin{abstract}
This paper investigates the control problem of magnetic levitation system, in which velocity feedback signal is influenced by stochastic disturbance. Firstly, single-degree-freedom magnetic levitation is regarded as an energy-transform action device. From the view of energy-balance relation, the magnetic levitation system is transformed into port-controlled Hamiltonian system model. Next, based on the Hamiltonian structure, the control law of magnetic levitation system is designed by applying Lyapunov theory. Finally, the simulation verifies the correctness of the proposed results.
\end{abstract}

\section{Introduction}

Magnetic levitation system is a class of typical nonlinear system, which is difficult to establish accurate mathematical model for the natural parameter of electromagnetic part dependent times $[1,2]$. Normally, a standard magnetic levitation system consists of four parts: the sensors, the controller, the power amplifier, and the electromagnetic drives. This kind of system is a control system and its control objective is to ensure that the soliquoid is in the normal position through a series of feedback and control activities. The general process is as follows: firstly, after comparing the given signal with the feedback signal, they will be passed along regulating circuit to power amplifier circuit; then the control currents flow through the power amplifier; finally, the electromagnet converts it to electromagnetic force to control the suspension position. Shu et al. in [3] introduced the working principle of the magnetic levitation system. Combined with the classical theory of dynamics and electromagnet, the nonlinear equations of motion of the system are derived by the general form of Lagrange equation, and after the linearization processing around the operating point, the state space description of the system is obtained.

In addition, the systems are always affected by stochastic disturbance in many practical control problems, which always lead to system instability [4]. Therefore, more and more scholars and experts pay attention to the research of stability and control of stochastic nonlinear systems and a lot of research achievements have been made that greatly promoted the development of the system research [5-9]. In [9], the nonlinear stochastic $\mathrm{H}_{\infty}$ control of Itô-type differential systems with all the state, control input, and external disturbance is studied and a sufficient condition is given for the finite/infinite horizon $\mathrm{H}_{\infty}$ control of such a system by means of Hamilton-Jacobi inequality. More recently, some progress has been made toward solving the stability analysis and controller design for stochastic Hamiltonian systems [1012]. Sun and Peng in [12] studied the robust adaptive control problem for a class of time-delay stochastic Hamiltonian systems. An uncertainty-independent adaptive control law is designed to guarantee that the closed-loop Hamiltonian system is robustly asymptotically stable in the mean square.

Due to the highly nonlinear characteristics of the system, the controller design problem will be solved based on Hamiltonian energy theory. In fact, the energy-based Hamiltonian system method has been widely used in practical systems control [11, 13-19]. Based on the Hamiltonian system theory, the port-controlled Hamiltonian dissipative model of magnetic levitation system was established in [19], by using the Hamiltonian function as the storage function and 
the controller was designed, which is simple and easy to implement. A key feature of the systems, which is useful for stability analysis and stabilization, is that Hamiltonian function in a port-Hamiltonian systems can be used as a Lyapunov function which brings great convenience.

As is known, the controllers and regulators of the systems are always unavoidably affected by stochastic disturbances, and the study of the controlled systems with stochastic disturbance is of practical significance. Different from what have been studied, this present paper deals with the controller design problem of the magnetic levitation system with stochastic disturbances. Current efforts have been made to dispose the control problem of the stochastic magnetic levitation system on the basis of Hamiltonian energy theory. We regard the magnetic suspension as the energy conversion device and then derive a mathematical model of the single degree of freedom stochastic magnetic levitation system from the point of energy balance, which is transformed into a portcontrolled Hamiltonian system. Consequently, the controller of the stochastic magnetic levitation system is designed. Finally, a simulation example is given to verify the validity of the results.

The rest of this paper is organised as follows. Section 2 provides the problem formulation, the Hamiltonian modeling process of the stochastic magnetic levitation systems, and some preliminaries. Section 3 gives the main results. A simulation example is worked out in Section 4 to illustrate the results. Section 5 draws the concluding remarks.

Notations. $\|\cdot\|$ stands for either the Euclidean vector norm or the induced matrix 2-norm. A function $f(x) \in \mathbb{C}^{2}$ means that $f(x)$ is a twice differentiable continuous function. The notation $X \geq Y$ (resp., $X>Y$ ), where $X$ and $Y$ are symmetric matrices, means that the matrix $X-Y$ is positive semidefinite (resp., positive definite). $\lambda_{\max }(P)$ $\left(\lambda_{\min }(P)\right)$ denotes the maximum (minimum) of eigenvalue of a real symmetric matrix $P$. Throughout the paper, the superscript " $T$ " stands for matrix transposition. In addition, for the sake of simplicity, we denote $\partial H / \partial x$ by $\nabla H$.

\section{Problem Description and Transformation}

The physical model of the magnetic levitation train system, which includes the concentrated mass of train carriages (together with the supporting magnet) suspended on the rigid lead rail is shown in Figure 1, where $m$ is the quality of train carriage (including the supporting magnet); $g$ is gravitational constant; $\theta_{M}$ is the gap between the supporting magnet and the guide rail; $\theta_{0}$ is the gap between the guide rail and the reference plane; $\theta$ is the distance between the supporting magnet and the reference plane; $\theta_{M}=\theta_{0}+\theta$; $L(\theta)$ is the self-inductance of magnetic coil, which depends on the gap $\theta ; i$ is the current flowing through magnet spool; $R$ is the coil resistance; $u$ is the voltage at both ends of the magnet spool.

By invoking Kirchoff's voltage law and Newtons second law, the dynamic equations of the magnetic levitation system

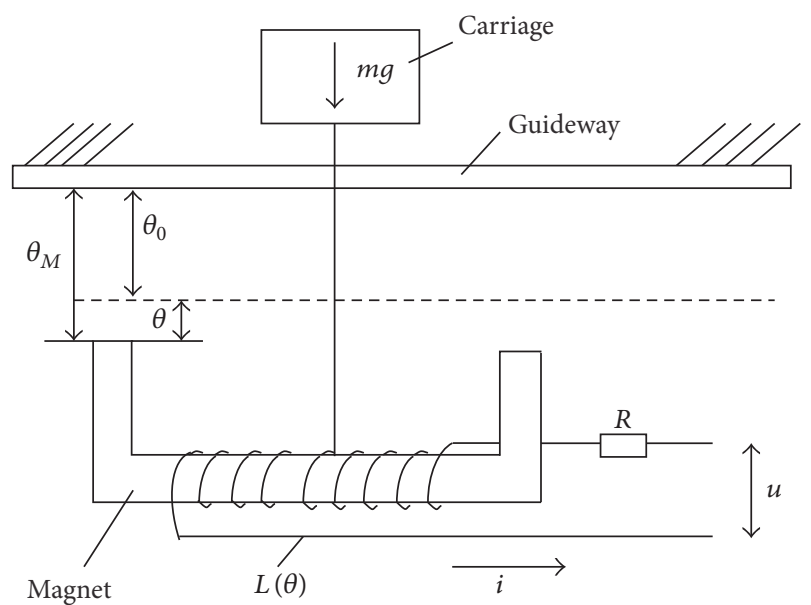

FIgURE 1: Physical model of magnetic suspension system.

can be obtained by taking the vertical upward direction as the positive direction:

$$
\begin{aligned}
m \ddot{\theta} & =F(i, \theta)-m g, \\
u & =R i+\dot{\Phi},
\end{aligned}
$$

where $\Phi=L(\theta) i$ is the magnetic flux and $F(i, \theta)$ is the force created by the electromagnet, which is given by

$$
F(i, \theta)=\frac{1}{2} \frac{\partial L}{\partial \theta}(\theta) i^{2}
$$

Here we regard the flux $\Phi$ as the independent variable; then (1) can be further transformed into the following forms:

$$
\begin{aligned}
\dot{\theta} & =v, \\
m \dot{v} & =\frac{\Phi^{2}}{4 k}-m g, \\
\dot{\Phi} & =u-R \frac{\Phi}{L(\theta)},
\end{aligned}
$$

where $k=\mu_{0} N^{2} S / 4, \mu_{0}$ is the permeability of vacuum, $N$ is coil turns, and $S$ is the effective pole area of the electromagnetic coil.

To obtain a port-controlled Hamiltonian model, we take a suitable approximation for the inductance is $L(\theta)=$ $2 k /\left(\theta_{M}-\theta\right)$. As is known, the speed of the rigid body will be affected by stochastic disturbances during the operation of the magnetic levitation system. Let $x=[\Phi, \theta, m \dot{\theta}]^{\mathrm{T}}=$ $\left[x_{1}, x_{2}, x_{3}\right]^{\mathrm{T}}$; due to the influence of stochastic disturbance, the magnetic levitation system (3) can be modeled as the following algebraic differential equations:

$$
\begin{aligned}
\mathrm{d} x_{1} & =-R \frac{x_{1}\left(\theta_{M}-x_{2}\right)}{2 k} \mathrm{~d} t+u \mathrm{~d} t+x_{3} \mathrm{~d} w(t), \\
\mathrm{d} x_{2} & =\frac{x_{3}}{m} \mathrm{~d} t, \\
\mathrm{~d} x_{3} & =\frac{x_{1}^{2}}{4 k} \mathrm{~d} t-m g \mathrm{~d} t,
\end{aligned}
$$


where $w(t)$ is an independent standard Wiener process and satisfies $E\{\mathrm{~d} w(t)\}=0$ and $E\left\{\mathrm{~d} w^{2}(t)\right\}=\mathrm{d} t$ and $E$ is the expectation operator. law as

The objective of this paper is to find a feedback control

$$
u(t)=\alpha(t)
$$

to ensure that the stochastic magnetic levitation system (4) with the controller (5) is asymptotically stable in the mean square.

Obviously, system (4) is a nonlinear system. In order to study the control problems of the stochastic magnetic levitation system in view of the energy balance, we need to convert it into a stochastic Hamiltonian system first. Taking the total of electromagnetic energy and mechanical energy as the Hamiltonian function, that is,

$$
H(x)=\frac{x_{1}^{2}}{4 k}\left(\theta_{M}-x_{2}\right)+\frac{1}{2 m} x_{3}^{2}+m g x_{2},
$$

then the magnetic levitation port-controlled Hamiltonian system is obtained:

$$
\mathrm{d} x(t)=(\mathbf{J}-\mathbf{R}) \nabla H(x) \mathrm{d} t+\mathbf{g}_{1} u \mathrm{~d} t+\mathbf{g}_{2}(x) \mathrm{d} w(t),
$$

where

$$
\begin{aligned}
\mathbf{J} & =\left(\begin{array}{ccc}
0 & 0 & 0 \\
0 & 0 & 1 \\
0 & -1 & 0
\end{array}\right), \\
\mathbf{R} & =\left(\begin{array}{lll}
R & 0 & 0 \\
0 & 0 & 0 \\
0 & 0 & 0
\end{array}\right), \\
\mathbf{g}_{1} & =\left(\begin{array}{l}
1 \\
0 \\
0
\end{array}\right), \\
\mathbf{g}_{2}(x) & =\left(\begin{array}{c}
x_{3} \\
0 \\
0
\end{array}\right) .
\end{aligned}
$$

According to the equilibrium condition of the system, the speed of the rigid body reduced to zero when the system is stable. Meanwhile, the electromagnetic force of the rigid body is equal to the gravity that acting upon on it. Then, we can get $x_{1}^{*}=\sqrt{4 k m g}, x_{3}^{*}=0$. Therefore the equilibrium point of the system is $x^{*}=\left[\sqrt{4 k m g}, x_{2}^{*}, 0\right]^{\mathrm{T}}$.

It is evident that $\mathbf{J}$ is a skew symmetric matrix, that is, $\mathbf{J}=$ $-\mathbf{J}^{\mathrm{T}}$, and $\mathbf{R}$ is a positive semidefinite matrix. Consequently, if $u=0$ and $w(t)=0$, system (7) is a dissipative Hamiltonian system since

$$
\mathscr{L} H(x)=-\nabla^{\mathrm{T}} H(x) \mathbf{R} \nabla H(x) .
$$

In order to design the controller of system (7), we introduce the following definition.
Definition 1. If there exists a controller $u$ such that

$$
\lim _{t \rightarrow \infty} \mathbb{E}\left\{\left\|x(t)-x^{*}\right\|^{2}\right\}=0,
$$

the stochastic Hamiltonian system (7) is said to be asymptotically stable in the mean square, where $x(t)$ is the solution of system (7) at time $t$ under the initial condition $x\left(t_{0}\right)=x_{0}$.

Next we introduce some auxiliary lemmas which will be used in this paper.

Lemma 2 (see [6]). For system

$$
\mathrm{d} x(t)=f(x(t)) \mathrm{d} t+g(x(t)) \mathrm{d} w(t), \quad \forall t \geq 0,
$$

assume that $f(x)$ and $g(x)$ are locally Lipschitz in $x$. For a constant $K>0$ and any $t$ satisfies $t \geq 0$, there exists function $V(x, t) \in \mathbb{C}^{2,1}\left(\mathbb{R}^{n} \times[0, \infty) ; \mathbb{R}_{+}\right)$such that

$$
\begin{aligned}
\mathscr{L} V & \leq K(1+V(x(t), t)), \\
\lim _{|x| \rightarrow \infty} \inf _{t \geq 0} V(x, t) & =\infty ;
\end{aligned}
$$

then from system (11) there exists a unique solution on $[0, \infty)$ for any initial date $x\left(t_{0}\right)=x_{0}$, where

$$
\begin{aligned}
\mathscr{L} V= & \frac{1}{2} \operatorname{tr}\left\{g^{\mathrm{T}}(x(t)) \frac{\left(\partial^{2} V\right)}{\partial x^{2}} g(x(t))\right\}+\frac{\partial V}{\partial t} \\
& +\frac{\partial V}{\partial x} f(x(t)) .
\end{aligned}
$$

Lemma 3 (see [4]). Let $V(x, t) \in \mathbb{C}^{2,1}\left(\mathbb{R}^{n} \times[0, \infty) ; \mathbb{R}_{+}\right)$; $\tau_{1}, \tau_{2}$ are the bounded stopping time and satisfy $0 \leq \tau_{1} \leq \tau_{2}$. If $V(x, t)$ and $\mathscr{L} V(x, t)$ are both bounded on $t \in\left[\tau_{1}, \tau_{2}\right]$, then

$$
\begin{gathered}
E\left\{V\left(x\left(\tau_{2}\right), \tau_{2}\right)-V\left(x\left(\tau_{1}\right), \tau_{1}\right)\right\} \\
=E \int_{\tau_{1}}^{\tau_{2}} \mathscr{L} V(x, t) \mathrm{d} t .
\end{gathered}
$$

Lemma 4. For any given matrices $A \in \mathbb{R}^{n \times r}$ and $G \in \mathbb{R}^{n \times n}$, if $G \geq 0$, it follows that

$$
\operatorname{tr}\left(A^{\mathrm{T}} G A\right) \leq \lambda_{\max }(G) \operatorname{tr}\left(A^{\mathrm{T}} A\right) .
$$

\section{Controller Design of Stochastic Magnetic Levitation System}

In this section, we will put forward the controller design scheme for stochastic magnetic levitation system (4). To this end, the stabilization problem of stochastic Hamiltonian system (7) is to be discussed first.

Consider system (7). Choose Lyapunov function as

$$
V(x)=H(x)-H\left(x^{*}\right) \geq 0 .
$$


Suppose that the Hamiltonian function $H(x) \in \mathbb{C}^{2}$ and satisfies

$$
\begin{aligned}
H(x) & \geq \alpha\left\|x-x^{*}\right\|^{2}, \\
\nabla^{\mathrm{T}} H(x) \nabla H(x) & \geq \beta\left\|x-x^{*}\right\|^{2},
\end{aligned}
$$

where $\alpha$ and $\beta$ are positive scalars. that

According to Itô differential equations, it can be obtained

$$
\mathrm{d} V(x)=\mathscr{L} V(x) \mathrm{d} t+\nabla V(x) \mathbf{g}_{2}(x) \mathrm{d} w(t)
$$

where

$$
\begin{aligned}
\mathscr{L} V(x)= & -\nabla^{\mathrm{T}} H(x) \mathbf{R} \nabla H(x) \\
& +\operatorname{tr}\left[\mathbf{g}_{2}^{\mathrm{T}}(x) \operatorname{Hess}(H(x)) \mathbf{g}_{2}(x)\right] \\
& +\nabla^{\mathrm{T}} H(x) g_{1} u .
\end{aligned}
$$

If we set suitable scalars $\lambda$ and $\mu$, then we have

$$
\begin{aligned}
\operatorname{tr} & {\left[\mathbf{g}_{2}^{\mathrm{T}}(x) \text { Hess }(H(x)) \mathbf{g}_{2}(x)\right] } \\
\leq & \frac{1}{2} \operatorname{tr}\left[\mathbf{g}_{2}^{\mathrm{T}}(x) \text { Hess }(H(x)) \text { Hess }^{\mathrm{T}}(H(x)) \mathbf{g}_{2}(x)\right] \\
& +\frac{1}{2} \operatorname{tr}\left[\mathbf{g}_{2}^{\mathrm{T}}(x) \mathbf{g}_{2}(x)\right] \\
\leq & \nabla^{\mathrm{T}} H(x)\left[\frac{1}{2}(\lambda+1) \mu I\right] \nabla H(x) .
\end{aligned}
$$

So the stabilization may be achieved by designing a suitable controller for system (7). The following theorem provides a feasible scheme.

Theorem 5 (consider system (7)). Suppose the Hamiltonian function $H(x)$ satisfies (17) and (18). Then, the closed-loop stochastic Hamiltonian system of (7) is asymptotic stable in the mean square under the feedback control law

$$
u=-\left[\mathbf{g}_{1}^{\mathrm{T}} \mathbf{g}_{1}\right]^{-1} \mathbf{g}_{1}^{\mathrm{T}}\left[\frac{1}{2}(\lambda+1) \mu I+I\right] \nabla H(x),
$$

where $\lambda$ and $\mu$ are scalars which satisfies $\lambda=$ $\sup _{t \geq 0}\|\operatorname{Hess}(H(x))\|^{2}$ and $\mu \geq \beta^{-1} \operatorname{tr}\left[\mathbf{g}_{2}^{\mathrm{T}}(x) \cdot \mathbf{g}_{2}(x)\right] \cdot\left\|x-x^{*}\right\|^{-2}$.

Proof. Substituting (22) into (7) yields

$$
\begin{aligned}
\mathrm{d} x(t)= & (\mathbf{J}-\mathbf{R}) \nabla H(x) \mathrm{d} t \\
& -\left[\frac{1}{2}(\lambda+1) \mu I+I\right] \nabla H(x) \mathrm{d} t \\
& +\mathbf{g}_{2}(x) \mathrm{d} w(t) .
\end{aligned}
$$

Combining (18), (20), (21), and (23), we obtain

$$
\mathscr{L} V(x) \leq-\nabla^{\mathrm{T}} H(x) \nabla H(x) \leq-\beta\left\|x-x^{*}\right\|^{2} ;
$$

then

$$
E\{\mathscr{L V}(x)\} \leq-\beta E\left\{\left\|x-x^{*}\right\|^{2}\right\}
$$

According to Lemma 3, the following formula was established: for all $t>0$,

$$
\begin{aligned}
E\{V(t)\}-E\{V(0)\} & =\int_{0}^{t} E\{\mathscr{L} V(s)\} \mathrm{d} s \\
& \leq \int_{0}^{t} E\left\{-\beta\left\|x(s)-x^{*}\right\|^{2}\right\} \mathrm{d} s .
\end{aligned}
$$

Therefore, the following formula was established:

$$
\frac{\mathrm{d}}{\mathrm{d} t} E\left\{\left\|x-x^{*}\right\|^{2}\right\} \leq-\frac{\beta}{\alpha} E\left\{\left\|x-x^{*}\right\|^{2}\right\} .
$$

Set $b=-\beta / \alpha$ and multiplying $e^{-b t}$ to the two sides of the inequality (27), we have

$$
e^{-b t} \frac{\mathrm{d}}{\mathrm{d} t} E\left\{\left\|x-x^{*}\right\|^{2}\right\}-e^{-b t} b E\left\{\left\|x-x^{*}\right\|^{2}\right\} \leq 0,
$$

that is,

$$
\frac{\mathrm{d}}{\mathrm{d} t}\left(e^{-b t} E\left\{\left\|x-x^{*}\right\|^{2}\right\}\right) \leq 0 .
$$

Integrating inequality (29) from $t_{0}$ to $t$, we get

$$
e^{-b t} E\left\{\left\|x-x^{*}\right\|^{2}\right\}-E\left\{\left\|x_{0}-x^{*}\right\|^{2}\right\} \leq 0,
$$

that is,

$$
E\left\{\left\|x-x^{*}\right\|^{2}\right\} \leq e^{b t} E\left\{\left\|x_{0}-x^{*}\right\|^{2}\right\}, \quad \forall t>0 .
$$

Since $b<0$, which implies that

$$
\lim _{t \rightarrow \infty} E\left\{\left\|x-x^{*}\right\|^{2}\right\}=0,
$$

system (7) is asymptotic stable in the mean square under the feedback control law (22). This completes the proof.

Remark 6. Since $H(x) \in \mathbb{C}^{2}, \mathbf{g}_{2}(x)$ are continuous functions and according to Lemma 2, we can conclude that the solution of the closed-loop system (23) is unique for any initial condition in the neighborhood of the equilibrium point $x^{*}$.

Next, we consider the stochastic magnetic levitation system (4). Obviously, there exist positive scalars $\alpha$ and $\beta$ which make system (4) meet the inequalities (17) and (18) in Theorem 5; thus we can get the following conclusions.

Theorem 7. The stochastic magnetic levitation system (4) is asymptotic stable in the mean square under the feedback control law

$$
u=-\left[\frac{1}{2}(\lambda+1) \mu+1\right] \frac{x_{1}}{2 k}\left(\theta_{M}-x_{2}\right),
$$

where $\lambda=\sup _{t \geq 0} \| \operatorname{Hess}\left(\left(x_{1}^{2} / 4 k\right)\left(\theta_{M}-x_{2}\right)+(1 / 2 m) x_{3}^{2}+\right.$ $\left.m g x_{2}\right) \|^{2}$ and $\mu$ is a scalar and satisfies $\mu \geq x_{3}^{2} /\left(\beta\|x\|^{2}\right)$.

Proof. Because of the stochastic magnetic levitation system (4) is equivalent to system (7), substitute $\mathbf{g}_{1}$ into system (7) and the formula (6) into (22); we can get controller (33). The rest of the proof is omitted here. 


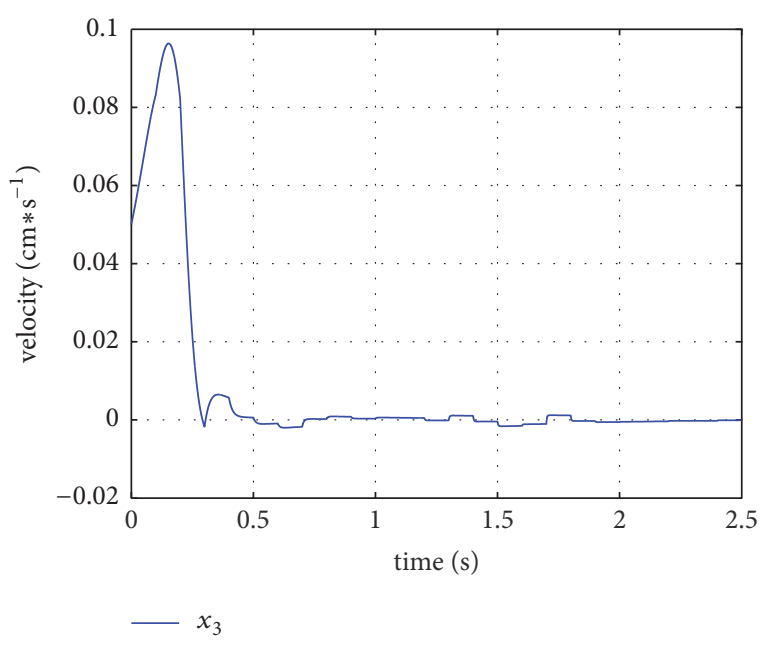

FIGURE 2: Velocity response curve.

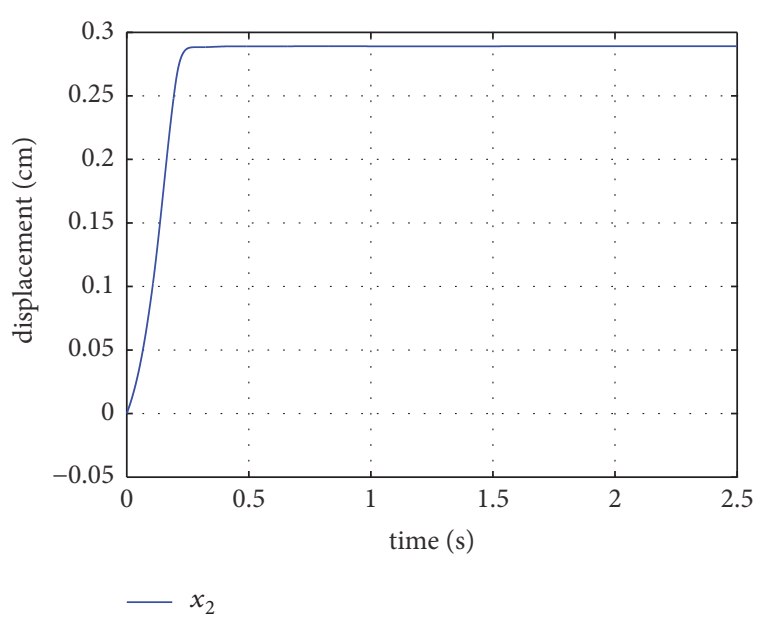

FIgURE 3: Displacement response curve.

\section{Illustrative Examples}

In this section, a simulation example is given to verify the correctness of the results obtained in this paper. The relevant parameters are given as follows: $R=4 \Omega, m=0.01 \mathrm{~g}, \theta_{M}=$ $0.01 \mathrm{~m}, k=0.05, g=0.0098 \mathrm{~N} / \mathrm{g}$, and $\theta_{0}=0.1 \mathrm{~cm}$. By calculating, we take $\lambda=100$ and $\mu=10$.

According to Theorem 7, we can see that system (4) is asymptotically stable in the mean square under the feedback control law

$$
u=-5060 x_{1}\left(\theta_{M}-x_{2}\right)
$$

The velocity curve of the rigid body is shown in Figure 2. It shows that the designed controller can make the system reach to the equilibrium point quickly. Figure 3 is the displacement curve of the rigid body; the displacement can also quickly reach to the equilibrium point.

\section{Conclusion}

This paper has investigated the control problem of stochastic magnetic levitation system. By regarding the magnetic levitation as the energy conversion device, we derived the mathematical model of single degree of freedom magnetic levitation system with stochastic disturbance from the point of view of the energy balance, and then the model can be transformed into a port-controlled Hamiltonian system. Then the controller of the stochastic magnetic levitation system has been designed based on the obtained Hamiltonian system model. Finally, the correctness of the conclusion has been verified by simulations. The main innovation of this paper is that we have fully taken into account the effect of random disturbances on the magnetic levitation system and solve the control problem under Hamiltonian systems framework by making full use of the dissipative structural properties of the Hamiltonian systems.

\section{Conflicts of Interest}

The authors declare that they have no conflicts of interest.

\section{References}

[1] L. Gentili and L. Marconi, "Robust nonlinear disturbance suppression of a magnetic levitation system," Automatica. A Journal of IFAC, the International Federation of Automatic Control, vol. 39, no. 4, pp. 735-742, 2003.

[2] F. Gómez-Salas, Y. Wang, and Q. Zhu, "Design of a discrete tracking controller for a magnetic levitation system: a nonlinear rational model approach," Mathematical Problems in Engineering, vol. 2015, Article ID 360783, 2015.

[3] G. Shu, W. Chen, and M. Reinhold, "The research on the model of a magnetic levitation system," Electric Machines and Control, vol. 9, no. 3, pp. 187-195, 2005 (Chinese).

[4] X. Mao and C. Yuan, Stochastic Differential Equations with Markovian Switching, Imperial College Press, London, UK, 2006.

[5] T. Li, G. Li, and Q. Zhao, "Adaptive Fault-Tolerant Stochastic Shape Control with Application to Particle Distribution Control," IEEE Transactions on Systems, Man, and Cybernetics: Systems, vol. 45, no. 12, pp. 1592-1604, 2015.

[6] S. Liu, S. Ge, and J. Zhang, "Adaptive output-feedback control for a class of uncertain stochastic non-linear systems with time delays," International Journal of Control, vol. 81, no. 8, pp. 1210$1220,2008$.

[7] L. Liu, X. Li, H. Wang, and B. Niu, "Global asymptotic stabilization of stochastic feedforward nonlinear systems with input time-delay," Nonlinear Dynamics. An International Journal of Nonlinear Dynamics and Chaos in Engineering Systems, vol. 83, no. 3, pp. 1503-1510, 2016.

[8] X. Wei, Z. Wu, and H. Karimi, "Disturbance observer-based disturbance attenuation control for a class of stochastic systems," Automatica. A Journal of IFAC, the International Federation of Automatic Control, vol. 63, pp. 21-25, 2016.

[9] W. Zhang, B. Chen, H. Tang, L. Sheng, and M. Gao, "Some remarks on general nonlinear stochastic $\mathrm{H}_{\infty}$ control with state, control, and disturbance-dependent noise," IEEE Transactions on Automatic Control, vol. 59, no. 1, pp. 237-242, 2014. 
[10] S. Satoh and K. Fujimoto, "Passivity based control of stochastic port-Hamiltonian systems," IEEE Transactions on Automatic Control, vol. 58, no. 5, pp. 1139-1153, 2013.

[11] W. Sun and L. Peng, "Observer-based robust adaptive control for uncertain stochastic Hamiltonian systems with state and input delays," Lithuanian Association of Nonlinear Analysts (LANA). Nonlinear Analysis. Modelling and Control, vol. 19, no. 4, pp. 626-645, 2014.

[12] W. Sun and L. Peng, "Robust adaptive control of uncertain stochastic Hamiltonian systems with time varying delay," Asian Journal of Control, vol. 18, no. 2, pp. 642-651, 2016.

[13] S. Knorn, A. Donaire, J. C. Aguero, and R. H. Middleton, "Passivity-based control for multi-vehicle systems subject to string constraints," Automatica, vol. 50, no. 12, pp. 3224-3230, 2014.

[14] R. Ortega, A. J. Van der Schaft, I. Mareels, and B. Maschke, "Putting energy back in control," IEEE Control Systems Magazine, vol. 21, no. 2, pp. 18-33, 2001.

[15] H. Ramrez, Y. Le Gorrec, B. Maschke, and F. Couenne, "On the passivity based control of irreversible processes: a portHamiltonian approach," Automatica, vol. 64, pp. 105-111, 2016.

[16] W. Sun, L. Peng, Y. Zhang, and H. Jia, " $\mathrm{H}_{\infty}$ excitation control design for stochastic power systems with input delay based on nonlinear Hamiltonian system theory," Mathematical Problems in Engineering, vol. 2015, Article ID 947815, 12 pages, 2015.

[17] W. Sun and B. Fu, "Adapative control of time-varying uncertain non-linear systems with input delay: a Hamiltonian approach," IET Control Theory \& Applications, vol. 10, no. 15, pp. 1844-1858, 2016.

[18] Y. Wang and S. S. Ge, "Augmented Hamiltonian formulation and energy-based control design of uncertain mechanical systems," IEEE Transactions on Control Systems Technology, vol. 16, no. 2, pp. 202-213, 2008.

[19] J. Zhang and J. Wu, "Hamiltonian modeling and passive control of magnetic levitation system," Dianji yu Kongzhi Xuebao/Electric Machines and Control, vol. 12, no. 4, pp. 464472, 2008. 


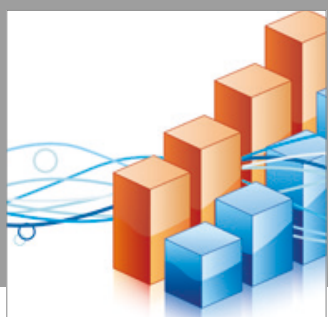

Advances in

Operations Research

vatersals

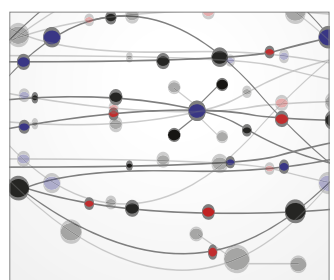

\section{The Scientific} World Journal
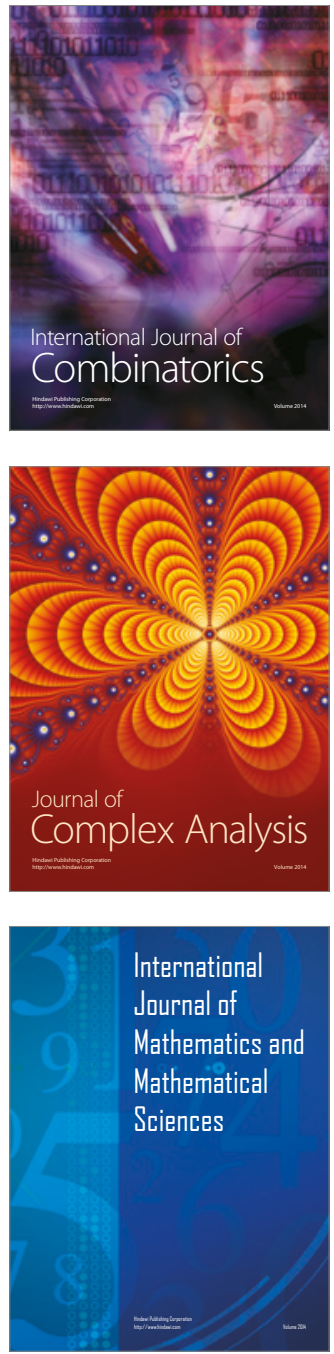
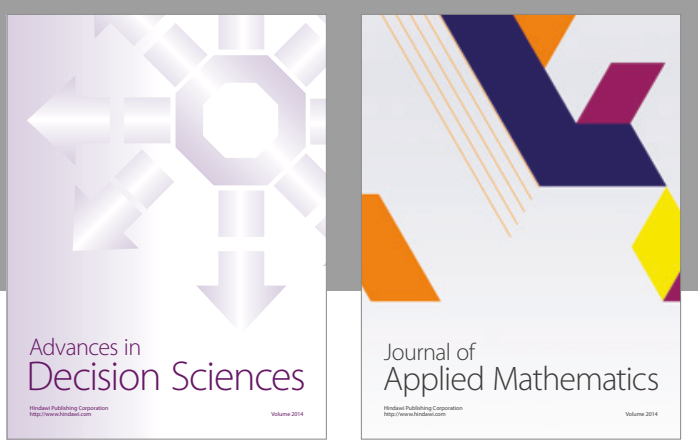

Algebra

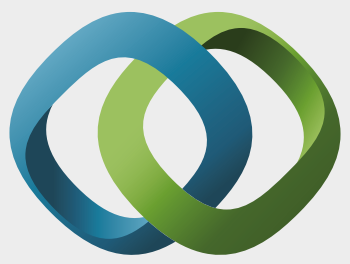

\section{Hindawi}

Submit your manuscripts at

https://www.hindawi.com
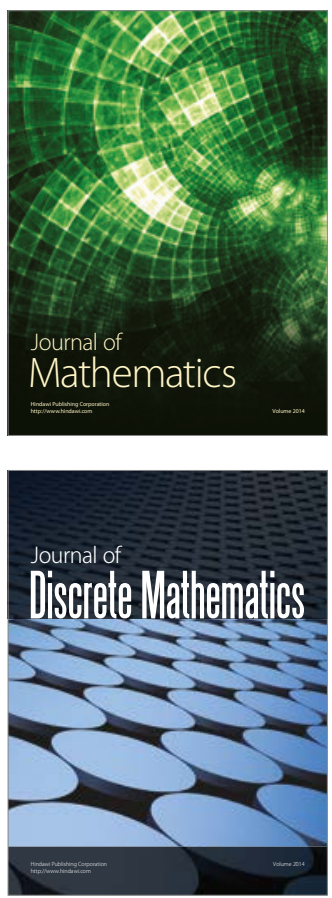

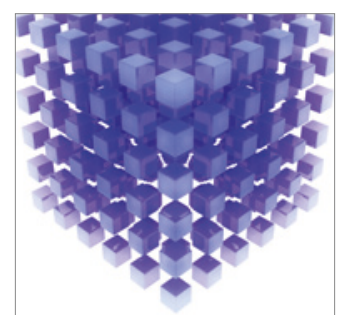

Mathematical Problems in Engineering
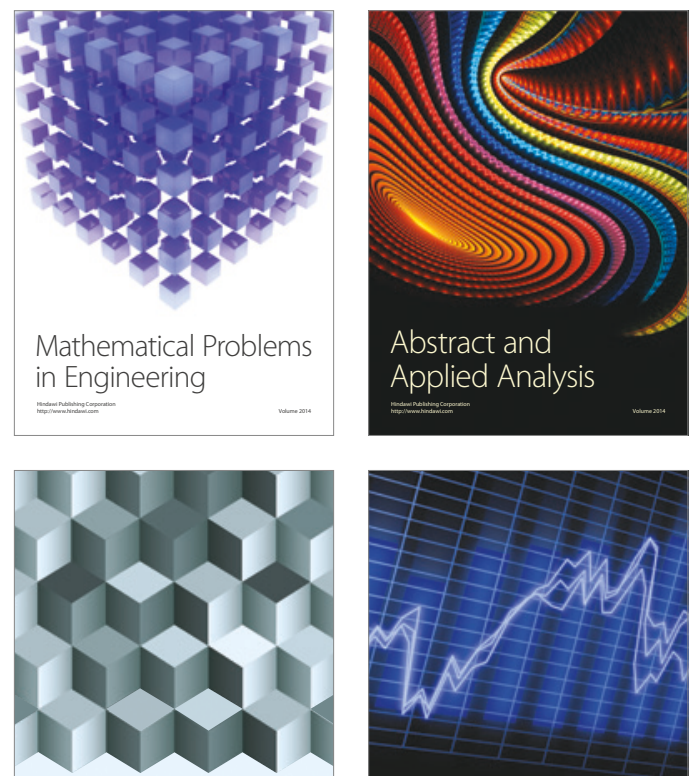

Journal of

Function Spaces

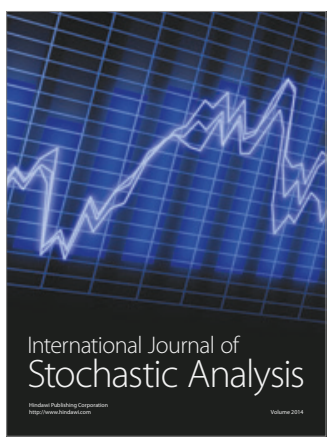

Probability and Statistics
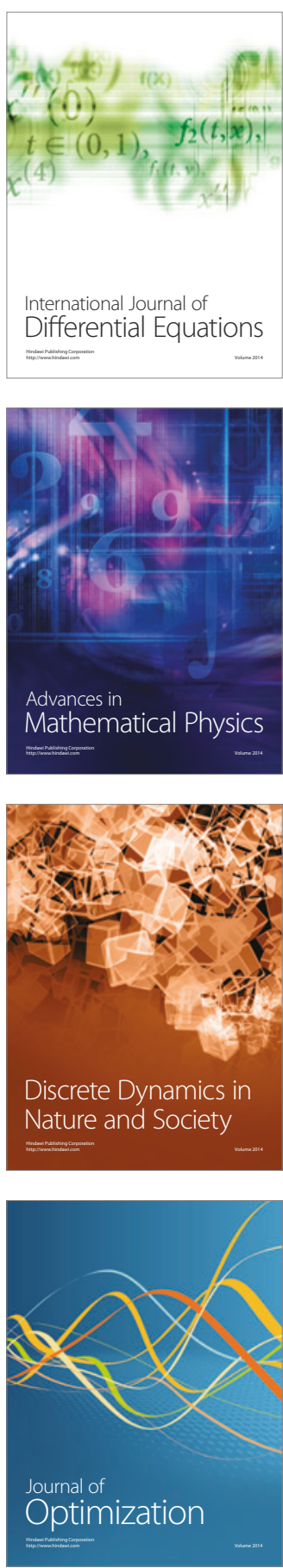